\section{Anything is Possible}

$\mathrm{R}$ ecently I had the pleasure of reading John Maxwell's little book, How Successful People Think [1]. Early in my reading, it was easy to grasp gems as I reflected upon my past four years as a Board member with Dietitians of Canada (DC). I continue to have an appreciation of the dedication that the staff, members, and Board of DC endlessly share, ensuring the advancement of our profession. All are truly "successful people" on so many accounts!

As professionals, we see things from another's point of view, enabling teamwork and looking beyond the immediate, while staying on target. This is a characteristic of "big picture" thinking and leadership — a key element as we work with clients, satisfy customers, help the less fortunate, etc.

Our visionary skill of strategic thinking, as exemplified by Vision 2020 , is proof of how we enable the most direct path toward achieving objectives in an era when many find it hard to plan their lives beyond one day. We need to embrace "possibility thinking" by looking for positive possibilities despite tough circumstances. In short, to achieve something, let yourself believe it is possible, no matter what experts might say.

"The value of life lies not in the length of days, but in the use we make of them; a man may live long yet live very little"-Michel Eyquem de Montaigne.

We can spend our lives any way we want, knowing we can only spend it once. Therefore, continue to surround yourself with "successful people" and you will be revolutionary.

(Can J Diet Pract Res. 2016;77:57)

(DOI: $10.3148 /$ cjdpr-2016-006)

Published at dcjournal.ca on 11 May 2016.

\section{Reference}

1. Maxwell, J. How successful people think: change your thinking, change your life. Nashville: Center Street, 2009.
ChaIR's Message

MESSAGE DE LA PRÉSIDENTE

\section{Tout est possible}

J

'ai récemment eu le plaisir de lire le court livre de John Maxwell intitulé How Successful People Think $<<$ Comment pensent les personnes qui réussissent $>>$ [1]. Tôt dans ma lecture, il a été facile pour moi de saisir la sagesse du propos alors que je réfléchissais à mes quatre dernières années à titre de membre du conseil d'administration des Diététistes du Canada (DC). Je continue d'apprécier le dévouement dont font inlassablement preuve le personnel, les membres et le conseil d'administration des DC, assurant ainsi l'avancement de notre profession. Tous ces gens sont des " personnes qui réussissent » à de très nombreux égards!

En tant que professionnels, nous percevons les choses du point de vue de l'autre, ce qui nous permet de favoriser le travail d'équipe et de nous projeter au-delà de l'immédiat, tout en restant fidèles à nos objectifs. Il s'agit là d'une façon d'envisager la situation dans son ensemble et de faire preuve de leadership - ce qui constitue un élément clé au moment de travailler avec des clients et de les satisfaire, de venir en aide aux plus démunis, etc.

Notre aptitude avant-gardiste à réfléchir de façon stratégique, comme en témoigne Vision 2020, prouve notre capacité à suivre le chemin le plus direct pour atteindre nos objectifs à une époque où bon nombre de gens ont du mal à planifier leur vie au-delà d'une journée. Nous devons adopter une " pensée qui n'écarte aucune possibilité » en tentant de trouver des possibilités positives, même en présence de circonstances difficiles. En somme, pour accomplir quelque chose, permettez-vous d'y croire, peu importe ce que les experts peuvent en dire.

"La valeur de la vie ne réside pas dans la durée, mais dans ce qu'on en a fait. Tel a vécu longtemps qui a pourtant peu vécu » (Michel Eyquem de Montaigne).

Sachant que nous n'avons qu'une vie à vivre, nous pouvons la vivre comme bon nous semble. Ainsi, continuez à vous entourer de "personnes qui réussissent » et vous serez révolutionnaire.

(Rev can prat rech diétét. 2016;77:57)

(DOI: $10.3148 /$ cjdpr-2016-006)

Publié au dcjournal.ca le 11 mai 2016.

\section{Référence}

1. Maxwell, J. How successful people think: change your thinking, change your life. Nashville: Center Street, 2009.

\section{5-2016 \\ Board of}

Directors

Conseil

d'administration
Phyllis Marsch (Chair)

Winnipeg, $\mathrm{MB}$

204-275-1918

Doug Cook

Toronto, ON

416-413-9095
Sarah Hewko

Edmonton, $\mathrm{AB}$

780-628-2080

Michelle McLearn

Middleton, NS

902-825-6160 Ext. 364
Nicole Spencer

Vancouver, BC

604-564-6534

Joane Routhier

Kirkland, QC

514-398-7749

$\begin{array}{ll}\text { Judy Sheeshka } & \text { Jean Helps } \\ \text { Surrey, BC } & \text { Headingly, MB } \\ \text { 778-294-3723 } & \text { 204-837-3166 }\end{array}$

Melissa Baker

Toronto, ON

647-881-4576
Chief Executive Officer

Marsha Sharp

416-642-9314 